\title{
Pedestrian-to-vehicle communication-based safety message transmission for the elderly in the conflict area
}

\author{
Woong Cho \\ Department of Computer System Engineering, \\ Jungwon University, \\ 85, Munmu-ro, Goesan-eup, Goesan-gun, \\ Chungbuk 28024, South Korea \\ Email:wcho@jwu.ac.kr
}

\begin{abstract}
Vehicular communications can be used for safety-related services by converging information and communication technology (ICT) with vehicles. In this paper, we consider pedestrian-to-vehicle (P2V) communication-based safety message transmission for the elderly. We focus on the conflict area that represents the unsigned area on the road, i.e., a single road and intersection area. Service scenarios for safety message transmission and the overall system architecture are introduced at first. Then, the overview of the communication and location information technologies for the services is introduced. After considering implementation issues for the suggested scenarios, we suggest an appropriate communication technology for P2V communication.
\end{abstract}

Keyword: P2V; pedestrian-to-vehicle communication; vehicular communication; elderly; conflict area; safety message.

Reference to this paper should be made as follows: Cho, W. (2016) 'Pedestrian-to-vehicle communication-based safety message transmission for the elderly in the conflict area', Int. J. Advanced Media and Communication, Vol. 6, No. 1, pp.57-64.

Biographical notes: Woong Cho received his $\mathrm{PhD}$ in Electrical and Computer Engineering from the University of Florida, Gainesville, in 2007. He was a Senior Research Engineer from February 2008 to February 2012 in Electronics and Telecommunications Research Institute (ETRI), Daejeon, South Korea. $\mathrm{He}$ is currently with the Department of Computer System Engineering in Jungwon University, Goesan, South Korea. His research interests are cooperative/relay networks, vehicular communications and signal processing for wireless communications.

\section{Introduction}

Vehicular communications provide various services by applying information and communication technology (ICT) to vehicle/road. One of the typical services using vehicular communications is safety-related application, where the services are mostly provided by either vehicle-to-vehicle $(\mathrm{V} 2 \mathrm{~V})$ communication or vehicle-to-infrastructure (V2I) communication (Bai and Krishnan, 2006; Biswas et al., 2006; Willke et al., 2009). 
On the other hand, pedestrian-to-vehicle (P2V) communication has also been considered for providing safety-related services. Prototype for P2V system using $3 \mathrm{G}$ wireless and wireless local area network (WLAN) was introduced in Sugimoto et al. (2008). Some field test results using P2V communication were introduced in Nagai et al. (2012), GM (2012), Honda (2013) and Lewandowski et al. (2013). In addition to practical tests, study on traffic safety at intersection was considered in Hu and Pei (2011), Xianglong and Jian (2011) and Anaya et al. (2014). However, they mostly consider safety-related service for general users and global positing system (GPS) is used for location estimation the existing work. It is indicated that GPS has $\sim 10 \mathrm{~m}$ of error even in good weather condition (Anaya et al., 2014). Therefore, GPS may not be sufficient to provide a precise location for the location constraint applications. Most of P2V systems adopt WLAN-based system due to its benefit of expendability and usability. In this paper, we consider P2V communication-based safety service for the elderly at the specific area, i.e., an unsigned a single road and intersection. We defined these areas as the conflict area in this paper. It is worth mentioning that IEEE $802.11 \mathrm{p}$ standard is widely used for exchanging data in V2V and V2I communications in general (IEEE Std P802.11p, 2010), whereas there is no dominant communication technology in $\mathrm{P} 2 \mathrm{~V}$ communication. We will overview various communication technologies to determine the specific communication technology for $\mathrm{P} 2 \mathrm{~V}$ communication for the proposed scenarios.

The rest of paper is organised as follows. The safety service scenarios and the overall system architecture are described at Sections 2 and 3, respectively. In Section 4, the overview of communication and location estimation technologies is discussed including implementation issues. The conclusions are given in Section 5.

\section{Safety service scenarios}

In this section, we introduce service scenarios. The target pedestrians for the safety message transmission are the elderly. Nowadays the rate of the elderly is increasing enormously in many countries including South Korea, and the death rate of the elderly due to traffic accident also increases. The death rate is 23 times larger than the children's one because of their decrement of physical function and overconfidence about surrounding environments. In South Korea, $\sim 47.3 \%$ of the death rate is the elderly among all age group while walking, and $\sim 80 \%$ of death accident occurs near/at the single road or intersection (Road traffic authority, 2011). Therefore, we focus on the safety application at the specific area, i.e., the conflict area.

Figure 1(a) and (b) represent service scenarios of safety message transmission at the single road and intersection, respectively. The system consists of on board unit (OBU), pedestrian unit (PU), road side unit (RSU) and Server/Controller. We assume that all pedestrians and vehicles are equipped with PU and OBU. RSU is installed besides the road which is represented as access point (AP) in Figure 1. All these units have communication device which can be used for exchanging safety message between units in the form of either pedestrian-to-infrastructure (P2I) or infrastructure-to-vehicle (I2V) communication. PU and OBU express the received information with alarm, light or vibration. Server/Controller stores and manages data from both pedestrians and vehicles. By combining P2I and I2V communications, communication links between pedestrians and vehicles are established. Therefore, $\mathrm{P} 2 \mathrm{~V}$ communication in this paper is not a directlink communication but a one-hop communication. 
Figure 1 Service scenarios: (a) single road and (b) intersection (see online version for colours)

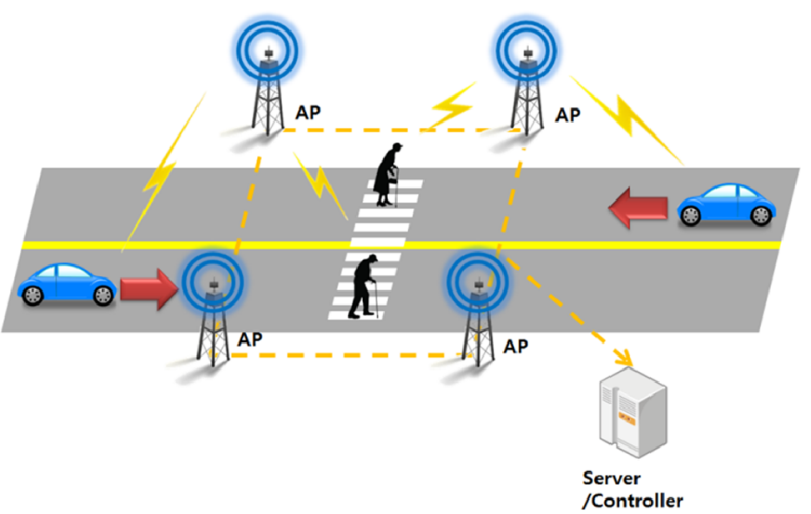

(a)

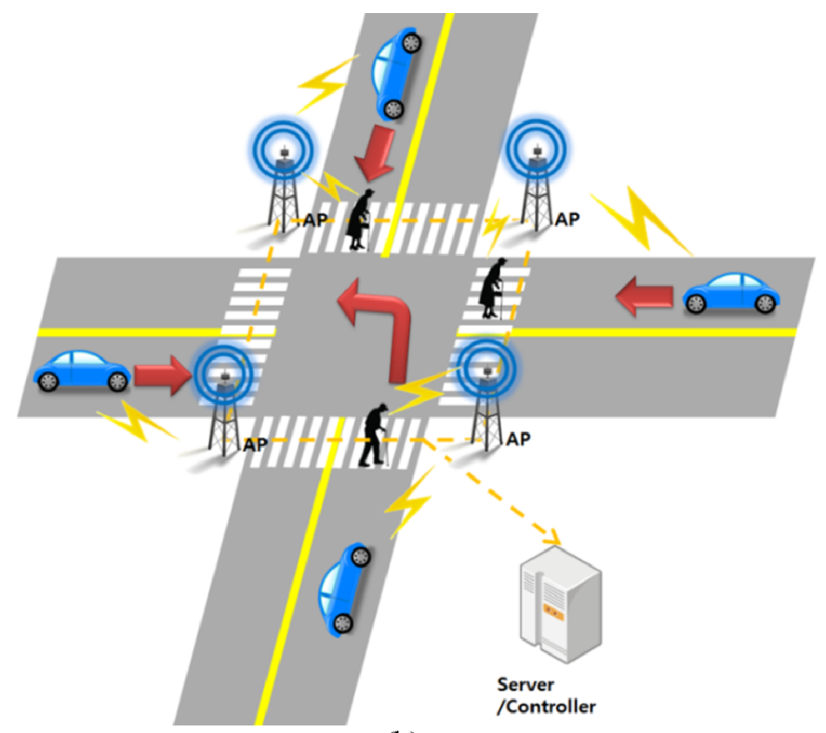

(b)

With this setup, communication scenarios between pedestrians and vehicles are divided two cases.

Case 1: Safety message transmission from vehicles to pedestrians.

1 Vehicle approaches AP(RSU).

2 Vehicle is located inside of AP's communication range.

3 AP detects a vehicle, and then vehicle's information (ID and location information) is transmitted to AP via V2I communication.

4 AP collects/manages pedestrians' information.

5 AP transmits safety message to pedestrian via I2P communication.

6 Pedestrians receive message and note that there is a vehicle near their location, which can avoid the accident due to the caution of pedestrians. 
Case 2: Safety message transmission from pedestrians to vehicles.

1 Pedestrian approaches AP(RSU).

2 Pedestrian is located inside of AP's communication range.

3 AP detects a pedestrian, and then pedestrian's information (ID and location information) is transmitted to AP via P2I communication.

4 AP collects/manages vehicles' information.

5 AP transmits safety message to vehicles via I2V communication.

6 Vehicles receive message and note that there is a pedestrian near their location, which can avoid the accident due to the caution of vehicles.

\section{System architecture}

More detailed data exchange flow focusing on communication link is depicted in Figure 2. From Figures 1 and 2, we can see that RSU and PU/OBU transmit and receive data by bi-directional communications. PU and OBU can express the received message with a certain form. We can also make it possible to PU/OBU communicates with smart phone. Then, the safety message can be delivered by smart phone also. Server/Controller plays an important role in the overall system. Server may store and delete the data from vehicles and pedestrians in the conflict area. These data are managed by the Controller. Controller calculates and decides which pedestrian/vehicle is valid for transmitting safety message for the corresponding vehicle/pedestrian. Therefore, AP data transmissions are managed by Controller. To do that Server/Controller is connected to AP and all Aps' data are collected by the Server/Controller. The data has to contain the information of each vehicle/pedestrian's own identification with location and moving direction. To estimate correct moving direction and distinguish sidewalk, we may require precise location information.

Figure 2 Data exchange flow between communication devices (see online version for colours)

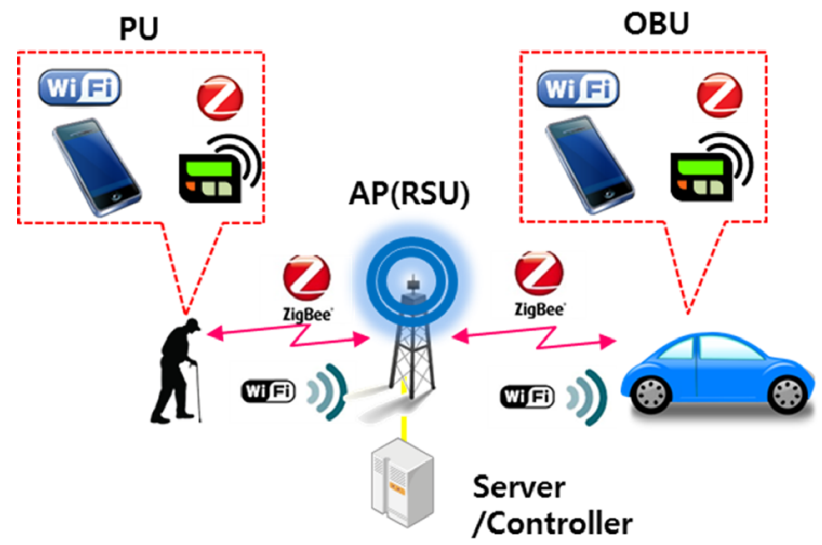




\section{Technical issues}

As we mentioned earlier, each device of vehicles, pedestrians and road exchanges data via communication. To establish communication link, a proper communication technology has to be chosen. In addition to communication technology, location information is also critical to indicate exact location of vehicles and pedestrians. In this section, we introduce some candidate communication and location information technologies for exchanging data.

\subsection{Overview of communication technologies}

There are various communication technologies in wireless communications. We consider IEEE $802.11 \mathrm{a} / \mathrm{b} / \mathrm{g}$ (WLAN), IEEE 802.15.4a and IEEE 802.11p (WAVE: Wireless Access in Vehicular Environments) since safety message transmission is provided a specific area. IEEE $802.11 \mathrm{a} / \mathrm{b} / \mathrm{g}$ is well-known technology which mainly provides internet service in the office, house and the area with APs. IEEE 802.15.4a is the modification of ZigBee (IEEE 802.15.4) by defining additional physical layers, i.e., chirp spread spectrum (CSS) and ultra wide band (UWB). IEEE 802.11p is a modified version of IEEE $802.11 \mathrm{a} / \mathrm{g}$ by changing some parts of physical and medium access layer. To determine proper communication technology, we first consider some requirements for the service, such as communication range, frequency usage, power consumption and etc. Table 1 compares communication technologies with respect to various items. In frequency band and frequency usage aspects, IEEE $802.11 \mathrm{a} / \mathrm{b} / \mathrm{g}$ and IEEE $802.15 .4 \mathrm{a}$ with CSS are useful since they use unlicensed band, i.e., industrial, scientific and medical (ISM) band. For the given scenarios, $\sim 250 \mathrm{kbps}$ of data rate is enough to transmit safety message. Therefore, all three technologies can be applied. Three technologies also satisfy the communication range constraint since the proposed system is implemented in the conflict area. Power consumption is directly related to battery life of device. For OBU and RSU, there is no limitation regarding power consumption. However, for PU, IEEE 802.15.4a is the best solution in terms of power consumption. It is worth noticing that link set up time and latency are also critical factors to transmit information in vehicular communication. However, in this paper, we only focus on the conflict area where vehicles are moving in relatively low speed, i.e., $\sim 50 \mathrm{~km} / \mathrm{h}$, which implies vehicles have enough time to set up and transmit data. Therefore, we omit time-related items in communication requirements.

\subsection{Overview of location information technologies}

Location information is important to provide the location of vehicles and pedestrians to each other. To determine the location of devices, two schemes are commonly applied. One is using global positioning system (GPS), the other is based on communication. For the system using GPS, there are three types of application, i.e., standalone GPS (S-GPS), assisted-GPS (A-GPS) and differential GPS (DGPS). S-GPS is only using satellite for estimating location. Whereas A-GPS and DGPS use the existing networks such as $3 \mathrm{G} / 4 \mathrm{G} / \mathrm{Wi}-\mathrm{Fi}$ and a fixed reference station for adjusting location information, respectively. The S-GPS has $\sim 10 \mathrm{~m}$ of accuracy. A-GPS is widely used in smart phone and has several metres of accuracy. The range of DGPS is several metres to $10 \mathrm{~cm}$ depending on the implementation technology. Although high accuracy can be achieved 
using the specific GPS, it requires additional systems, which causes increment of cost. The drawback of GPS-based system is the location information may be lost when line-of-sight (LOS) is not guaranteed.

Table 1 Comparison of communication technologies

\begin{tabular}{lccc}
\hline Item & IEEE $802.11 \mathrm{a} / \mathrm{b} / \mathrm{g}$ & IEEE $802.15 .4 a$ & IEEE $802.11 \mathrm{p}$ \\
\hline Frequency band & $2.4 \mathrm{GHz}$ or $5.8 \mathrm{GHz}$ & UWB $(250 \sim 750 \mathrm{MHz}$, & $5.85 \sim 5.925 \mathrm{GHz}$ \\
& & $3244 \sim 4742 \mathrm{MHz}$, & \\
& & $5944 \sim 10234 \mathrm{MHz})$ & \\
Frequency usage & Unlicensed & CSS: $2.4 \mathrm{GHz}$ & \\
& & UWB: various & Licensed \\
Bandwidth & $20 \mathrm{MHz}$ & CSS: Unlicensed & \\
& & UWB: various & $10 \mathrm{MHz}$ \\
Data rate & $54 \mathrm{Mbps}(\mathrm{Max})$ & CSS: $5 \mathrm{MHz}$ & \\
& & UWB: Various & $27 \mathrm{Mbps}(\mathrm{Max})$ \\
Communication range & $200 \mathrm{~m}$ & CSS: $1 \mathrm{Mbps}$ & \\
Power consumption & Small & $100 \mathrm{~m}$ & $1 \mathrm{~km}$ \\
\hline
\end{tabular}

For the communication-based system, there are several methods of application, i.e., angle of arrival (AOA), time of arrival (TOA)/time difference of arrival (TDOA), received signal strength (RSS) and hybrid method. The location accuracy in communication-based system highly depends on the communication technology in addition to the location estimation algorithms. IEEE $802.11 \mathrm{a} / \mathrm{b} / \mathrm{g}$ and IEEE $802.11 \mathrm{p}$-based systems provide approximately several metres of accuracy. IEEE 802.15.4a can support 1-2 m of accuracy by modifying physical layer of IEEE 802.15.4 (Salman et al., 2010). However, to implement location estimation scheme, it is necessary to use some reference devices such as APs.

In summary, two schemes for estimating location are compared in Table 2. SGPS and A-GPS can be used in the open area with low cost and medium accuracy. For high accuracy using DGPS, we need very high cost with additional implementations. Communication-based systems can be applied in a specific area depending on the special purpose of applications.

Table 2 Comparison of location estimation scheme

\begin{tabular}{lcc}
\hline Item & GPS based & Communication based \\
\hline Properties & Can apply open area with free & Can apply specific area. \\
& Can achieve very high accuracy & \\
Typical accuracy & $\sim 10 \mathrm{~m}$ & $\sim 10 \mathrm{~m}$ \\
Max accuracy & $10 \mathrm{~cm}$ & $1 \sim 2 \mathrm{~m}$ \\
$\begin{array}{l}\text { Advantage and } \\
\text { disadvantage }\end{array}$ & LOS has to be guaranteed & Need additional APs \\
\hline
\end{tabular}




\subsection{Implementation issue}

To realise practical system, we have to choose a proper communication technology with location estimation scheme. Our scenarios mainly focus on the conflict area with vehicles and pedestrians (the elderly) carrying communication devices. Since safety message transmission is closely related to public safety, everybody can use the system. Then, ISM band is a good choice in terms of frequency usage. Approximately $100 \mathrm{~m}$ of communication range is enough in the conflict area, and we do not need high data rate for message transmission. For PU, it is better to choose the device with low power consumption. For location information aspect, communication-based system with high accuracy $(<2 \mathrm{~m})$ is good candidate, since the safety service is applied a specific area, and road and sidewalk is clearly separated. Based on these analyses, we can conclude IEEE 802.15.4a is a good candidate for safety message transmission among the aforementioned communication technologies.

\section{Conclusions}

In this paper, we introduced safety message transmission systems for the elderly that can be applied in the conflict area. Service scenarios with overall system architecture are considered. After discussing technical issues in communication and location information technologies, we proposed a specific communication technology. In the future, it is needed to verify the performance of the proposed systems by adopting suggested technology.

\section{Acknowledgements}

This work was supported by the Jungwon University Research Grants.

\section{References}

Anaya, J.J., Merdrignac, P., Shagdar, O., Nashashibi, F. and Naranjo, J.E. (2014) 'Vehicle to pedestrian communications for protection of vulnerable road users', IV 2014: Proceedings of Intelligent Vehicles Symposium, Dearborn, MI, USA, pp.1037-1042.

Bai, F. and Krishnan, H. (2006) 'Reliability analysis of DSRC wireless communication for vehicle safety applications', ITSC 2006: Proceedings of the IEEE Intelligent Transportation Systems Conference, Toronto, Ont. Canada, pp.355-362.

Biswas, S., Tatchikou, R. and Dion, F. (2006) 'Vehicle-to-vehicle wireless communication protocols for enhancing highway traffic safety', IEEE Communications Magazine, Vol. 44, No. 1, pp.74-82.

GM (2012) http://www.wired.com/2012/07/gm-wifi-pedestrians/ (Accessed 27 July, 2012).

Honda (2013) http://articles.sae.org/12408/ (Accessed 30 September, 2013).

Hu, L. and Pei, Y. (2011) 'Traffic safety assessment between pedestrian-automobile near intersection', TMEE 2011: Proceedings of the International Conference on Transportation, Mechanical, and Electrical Engineering, Changchun, China, pp.1788-1791.

IEEE Std P802.11p (2010) IEEE Standard for Information Technology-Telecommunications and Information Exchange Between Systems-Local and Metropolitan Area Networks-Specific Requirements, Part 11, Amendment 6: Wireless Access in Vehicular Environments. 
Lewandowski, A., Böcker, S., Köster, V. and Wietfeld, C. (2013) 'Design and performance analysis of an IEEE 802.15.4 V2P pedestrian protection system', WiVeC 2013: Proceedings of International Symposium on Wireless Vehicular Communications, Dresden, Germany, pp.1-6.

Nagai, M., Hakaoka, K. and Doi, Y. (2012) 'Pedestrian-to-vehicle communication access method and field test results', ISAP.2012: Proceedings of 2012 International Symposium on Antennas and Propagation, Nagoys, Japan, pp.712-715.

Road Traffic Authority (2011) Statistical Analysis of Traffic Accidents, 2012-0225-052, 2012.

Salman, N., Rasool, I. and Kemp, A.H. (2010) 'Overview of the IEEE 802.15.4 standard family for low rate wireless personal area networks', ISWCS 2014: Proceeding of International Symposium on Wireless Communication Systems, York, UK, pp.701-705.

Sugimoto, C., Nakamura, Y. and Hashimoto, T. (2008) 'Prototype of pedestrian-to-vehicle communication system for the prevention of pedestrian accidents using both $3 \mathrm{G}$ wireless and WLAN communication', ISWPC 2008: Proceedings of International Symposium on Wireless Pervasive Computing, pp.764-767.

Willke, T.L., Tientrakool, P. and Dion, F. (2009) 'A survey of inter-vehicle communication protocols and their applications', IEEE Communications Surveys and Tutorials, Vol. 11, No. 2, pp.3-20.

Xianglong, S. and Jian, L. (2011) 'Study on the pedestrian-vehicle safety based on traffic conflict at uncontrolled intersection', ICETCE 2011: Proceedings of 2011 International Conference on Electric Technology and Civil Engineering, pp.1614-1616. 